\title{
Malformaciones pulmonares congénitas
}

\section{Congenital lung malformations}

\author{
Dra. Verónica Giubergia \\ Pediatra Neumonóloga \\ Jefe de Clínica, Servicio de Neumonología \\ Hospital de Pediatría Juan P. Garrahan \\ Buenos Aires, Argentina
}

\begin{abstract}
CONGENITAL LUNG MALFORMATIONS
Congenital lung malformations (CLM) comprise a heterogeneous group of lung diseases. They vary widely in their clinical presentation and severity, depending on the degree of lung involvement and their location in the thoracic cavity. They can manifest at any age and can be the source of significant morbidity and mortality in infants and children. Patients with CLM can present respiratory symptoms at birth or can remain asymptomatic for long periods. There has been an increase in early diagnosis of CLM attributable to the routine use of prenatal ultrasound. Management of these lesions depends on the type of malformation and symptoms. T reatment of asymptomatic patients is controversial, because the prognosis of these diseases is unpredictable. Because of the risk of complications, most authors recommend resection of the lesion at the time of diagnosis. This review describes the principal CLM, their diagnosis, and the controversies regarding treatment.
\end{abstract}

Key words: Respiratory system abnormalities, congenital cystic adenomatoid malformation of lung, bronchopulmonary sequestration, congenital lobar emphysema, pulmonary hypoplasia.

\section{RESUMEN}

Las malformaciones pulmonares congénitas (MPC) constituyen un grupo heterogéneo de enfermedades. Varían en su presentación clínica y gravedad en función al grado de afectación pulmonar y a su ubicación en tórax. Se pueden manifestar a cualquier edad y pueden condicionar morbilidad y mortalidad significativa en lactantes y niños. Los pacientes pueden presentar síntomas respiratorios desde el nacimiento o permanecer asintomáticos durante largos períodos. Se ha observado un aumento en el diagnóstico precoz de las MPC atribuible al uso rutinario de la ecografía prenatal. El manejo de estas lesiones depende del tipo de malformación y de los síntomas. El tratamiento de pacientes asintomáticos es controvertido debido a que el pronóstico de estas enfermedades es desconocido. La mayoría de los autores recomiendan la resección de la lesión en el momento del diagnóstico debido al riesgo de complicaciones. Esta revisión describe las principales malformaciones congénitas del pulmón, su diagnóstico y las estrategias de tratamiento.

\section{INTRODUCCIÓN}

Las malformaciones pulmonares congénitas (MPC) conforman un grupo de entidades originadas en defectos del crecimiento broncopulmonar durante los distintos estadios evolutivos del pulmón. Para una comprensión más acabada es fundamental el conocimiento de las 6 etapas (las 4 primeras intraútero y las últimas 2 postnatales) en que se divide el desarrollo pulmonar normal.

- Período embrionario: entre la $4^{\mathrm{a}}$ y la $7^{\mathrm{a}}$ semana de gestación se desarrolla un divertículo ventral (respiratorio) en el extremo superior del intestino primitivo anterior. Al final de la $5^{\text {a }}$ semana las vías aéreas destinadas a convertirse en los cinco bronquios lobares han comenzado su desarrollo.

- Período pseudoglandular: (final de la $5^{\mathrm{a}}$ hasta la $17^{\mathrm{a}}$ semana de gestación). Se produce la ramificación de los bronquios en forma dicotómica que culmina con la formación de los bronquios terminales.

\section{Correspondencia: \\ Dra. Verónica Giubergia. \\ Dirección: Combate de los Pozos 1881 (1245) \\ Ciudad de Buenos Aires. Argentina. \\ Teléfono: +5491143081998 \\ Dirección de mail: verogiubergia@gmail.com}

ISSN 0718-33X. Derechos reservados.
- Período canalicular: (entre semanas 17 y 26). Se desarrolla la porción periférica del árbol bronquial. Se desarrollan los futuros bronquios respiratorios y se vasculariza el tejido pulmonar adyacente.

- Período sacular: comienza el desarrollo alveolar. Hacia la semana 26 el pulmón presenta racimos terminales de vías aéreas Ilamadas sáculos.

- Período alveolar: aparecen septos secundarios en los septos primarios existentes en el pulmón sacular.

- Maduración microvascular: la doble capa capilar de los septos alveolares inmaduros se reduce a una sola capa capilar.

En cada una de las etapas una variedad de señales reguladoras, incluyendo interacciones epitelio-mesenquimatosas, tensión de P02 fisiológica, fuerzas mecánicas así como factores humorales intervienen en el normal desarrollo del pulmón y de la vía aérea. Las MPC son el resultado de perturbaciones en la embriogénesis del pulmón y vías respiratorias. Tanto el lugar (nivel en el árbol traqueobronquial) como el momento (edad gestacional) del insulto embriológico se correlacionan con el tipo de lesión y la histopatología que se manifiesta. También se ha propuesto que una atresia bronquial con displasia pulmonar 
secundaria puede ser la condición patológica subyacente común a muchas lesiones congénitas representando una secuencia de obstrucción/malformación (1-4).

\section{DEFINICIÓN}

Se han establecido criterios clínicos, radiológicos e histopatológicos que permiten la clasificación de la mayor parte de las MPC, aunque como existen superposiciones puede resultar dificultosa. Aún no hay consenso en su nomenclatura. Algunos autores evitan el uso de los términos "malformación congénita" ya que consideran que son sinónimos. Otros utilizan los términos "malformaciones pulmonares de tórax" de forma más genérica. "MPC" es la denominación más empleada en la literatura y será utilizado en la presente revisión $(2,5,6)$.

Las MPC consideradas comprenden la malformación adenomatosa quística, el secuestro pulmonar, el quiste broncogénico y el enfisema lobar congénito. Otras MPC también consideradas son la agenesia, aplasia y la hipoplasia pulmonar.

Las MPC son poco frecuentes comparadas con las enfermedades respiratorias adquiridas, constituyendo entre el 8 y el $18 \%$ de todas las malformaciones (6).

En los últimos años se incrementó el diagnóstico precoz de las MPC directamente relacionado al uso rutinario de la ecografía prenatal durante el control del embarazo. Se estima que el $10 \%$ de los casos se identifican en el nacimiento, mientras que el $14 \%$ son diagnosticados a los 15 años de edad (7). En ocasiones, constituyen un hallazgo en una radiografía de tórax (RXT).

\section{AGENESIA, APLASIA E HIPOPLASIA PULMONAR}

Son MPC que se producen entre el día 26 y la semana 24 de gestación. Existen 3 grados de desarrollo interrumpido:

\section{Agenesia pulmonar}

Se observa la ausencia completa de uno o ambos pulmones sin rastros de irrigación vascular ni de formación de bronquios 0 de parénquima pulmonar. Las izquierdas tienen peor pronóstico con $50 \%$ de mortalidad en el período neonatal. La RXT muestra desviación del mediastino hacia el lado de la agenesia. El diagnóstico se confirma por tomografía de tórax (TC) y broncoscopía que evidencian la ausencia del parénquima y del bronquio fuente respectivamente.

\section{Aplasia pulmonar}

Existe una supresión de todas las estructuras bronquiales excepto por un bronquio rudimentario que termina en un saco ciego. No se puede determinar una diferenciación etiológica, patogénica o clínica entre agenesia y aplasia y deberían considerarse en forma conjunta.

\section{Hipoplasia pulmonar (HP)}

Se observa una disminución de la cantidad y/o tamaño de las vías aéreas, vasos y alvéolos. Puede ser asintomática y compatible con la vida o presentar dificultad respiratoria severa y estar acompañada de otras malformaciones. La HP raramente es primaria o idiopática. La compresión de pulmón extrínseca 0 intrínseca es una condición subyacente. Los hallazgos clínicos dependen del grado de anormalidad pulmonar y de la presencia de otras malformaciones congénitas. En el 50\% de los casos suele asociarse con hernia diafragmática, malformaciones genitourinarias, cardíacas y gastrointestinales. La hipertensión pulmonar persistente es un evento grave que puede presentarse en los recién nacidos con HP (3).

En los pacientes asintomáticos el examen físico revela una asimetría en la auscultación con ausencia de entrada de aire en el lado afectado. La radiografía de tórax (RXT) muestra un desplazamiento cardíaco con falta de aireación pulmonar del mismo lado. En la mayoría de los casos el pulmón contralateral muestra hiperinsuflación y desplazamiento hacia el hemitórax opuesto. La angiotomografía evidencia ausencia de rama pulmonar con ausencia total o parcial del parénquima pulmonar. El centellograma ventilación/perfusión muestra una disminución en la captación con disminución de la perfusión de la arteria pulmonar que en general es de menor calibre. La endoscopía corrobora el diagnóstico al evidenciar una reducción del calibre del bronquio hipoplásico.

El tratamiento es conservador. En aquellos pacientes con episodios de infección recurrente puede considerarse cirugía.

\section{MALFORMACIÓN ADENOMATOSA QUÍSTICA (MAQ)}

Es la MPC más frecuente que requiere tratamiento quirúrgico. Fue descripta por pimera vez en $1949(8,9)$. La incidencia es de aproximadamente 1 caso por cada 25.000-35.000 embarazos (6). Representa el $95 \%$ de todas las MPC quísticas. Una noxa entre las semanas de 5 y 22 de gestación podría condicionar su desarrollo.

Las lesiones varían ampliamente en tamaño y pueden afectar a un lóbulo entero o un segmento, así como involucrar todo un pulmón. Pueden ser bilaterales, aunque ocurre más frecuentemente en los lóbulos inferiores. El 85-95\% de los casos ocurre en un solo lóbulo $(6,8)$.

La definición y clasificación de estas lesiones siempre ha sido problemática. Stocker las clasificó en cinco tipos basados en el tamaño de los quistes postnatales y en la histología (10). Posteriormente se propuso una clasificación basada en la ecografía prenatal: lesiones macroquísticas (quistes mayores de $5 \mathrm{~cm}$ ) y microquísticas (quistes de $5 \mathrm{~mm}$ ).

- Tipo 0: se origina a partir de la tráquea o tejido bronquial con una baja incidencia (1-3\%). Los quistes son de hasta $0.5 \mathrm{~cm}$ de diámetro cubiertos por epitelio ciliado, pseudoestratificado. Afecta todos los lóbulos pulmonares y en general es incompatible con la vida. Se asocia con anomalías cardíacas graves. 
- Tipo I: es la más frecuente (50-70\%) y tiene el mejor pronóstico. Presenta un quiste único grande $(10 \mathrm{~cm})$ o múltiples mayores de $2 \mathrm{~cm}$. (Figura 1). Está conformada por epitelio ciliado, pseudoestratificado con áreas de epitelio secretor cubierto por tejido fibromuscular, generalmente llenas de aire o moco.

El tejido pulmonar adyacente normal está comprimido en general por la malformación, que tiene el efecto de una masa intratorácica y puede producir insuficiencia respiratoria en el período neonatal. Los síntomas se presentan en los primeros meses de vida. Ocasionalmente permanecen asintomáticos hasta la edad adulta.

- Tipo II: es el segundo grupo más frecuente (20-40\%). Presenta múltiples quistes de 0.5 a $2 \mathrm{~cm}$ que semejan bronquíolos terminales cubiertos por epitelio ciliado, cuboidal columnar. Afectan sólo un lóbulo. Están asociadas a agenesia renal, defectos cardiovasculares, hernia diafragmática, siringomielia, pectus y atresia de esófago que pueden condicionar su pronóstico. La asociación con secuestro pulmonar se observa hasta en un $40 \%$ de los casos (8).

- Tipo III: 10\% de los casos. Se trata de una masa sólida que desplaza el mediastino. La histología muestra estructuras milimétricas similares a bronquíolos cubiertos por epitelio ciliado, cuboidal. El efecto de masa de este tipo de MAQ puede causar desplazamiento del mediastino en el feto y provocar HP contralateral e hidrops fetal. (11).

- Tipo IV: 10-15\% de los casos. A menudo son erróneamente clasificados como de tipo I (10). Tiene un origen acinar distal con grandes quistes periféricos de hasta $7 \mathrm{~cm}$ de diámetro. La etiología es poco clara y es se ha propuesto como una etapa evolutiva del blastoma pleuropulmonar tipo 1, indistinguible en la imágenes de este tipo de MAQ (6).

En las MAQ la RXT suele mostrar múltiples quistes, de paredes finas llenos de aire o con contenido. Se puede observar también desplazamiento mediastínico y efecto de la masa. La TC determina el tamaño de los quistes, revela anomalías asociadas, define la extensión anatómica de la malformación y muestra lesiones que en las RXT o en la ecografía pueden no visualizarse $(6,8,12)$. El diagnóstico diferencial incluye los neumatoceles, abscesos, quiste broncogénico, secuestro pulmonar, enfisema lobar congénito y masas del mediastino (11) (Figura 1).

La ecografía prenatal evidenciar una área multiquística en el tórax. La resonancia magnética fetal (RMF) es útil en los casos en los que el diagnóstico no es claro (13). Se debe plantear el diagnóstico diferencial con hernia diafragmática.

Existe un amplia gama de formas evolutivas que pueden ser graves (hidrops fetal o HP con muerte intrauterina o perinatal) 0 tan leves como lesiones asintomáticas o inclusive resolución espontánea de las lesiones, como se ha observado hasta en un $15-50 \%$ de los casos probablemente relacionado a un mecanismo de apoptosis y supresión del flujo vascular) $(2,6,11,14)$.
El 90\% de los individuos con MAQ están asintomáticos en el período neonatal (6). Cuando la cirugía no se efectúa precozmente existe el riesgo de desarrollar infecciones pulmonares recurrentes. Asimismo, se han reportado casos de malignización (blastomas, carcinomas epidermoide 0 rabdomiosarcomas) por lo que ante la sospecha clínica deberá siempre efectuarse cirugía resectiva (8).

\section{Figura $\mathrm{N}^{\mathbf{0}} \mathbf{1}$}

Malformación adenomatosa quística tipo I: Iesión multiquística en lóbulo inferior derecho.

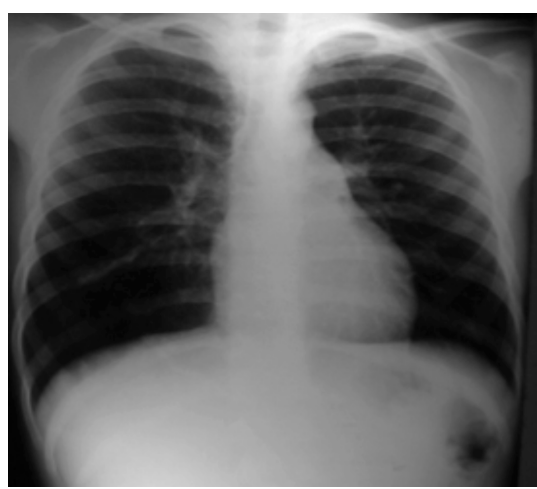

1 A. Radiografía de tórax.

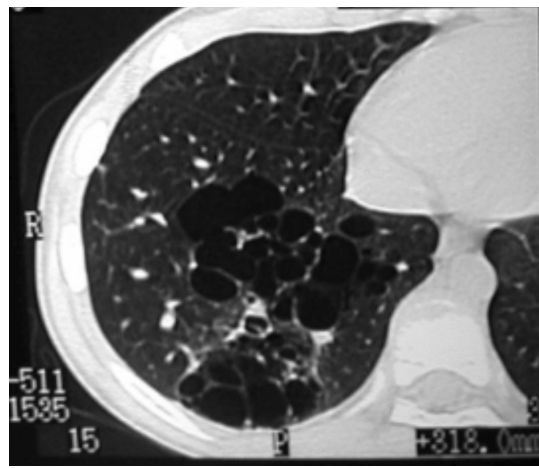

1 B. Tomografía computarizada de tórax

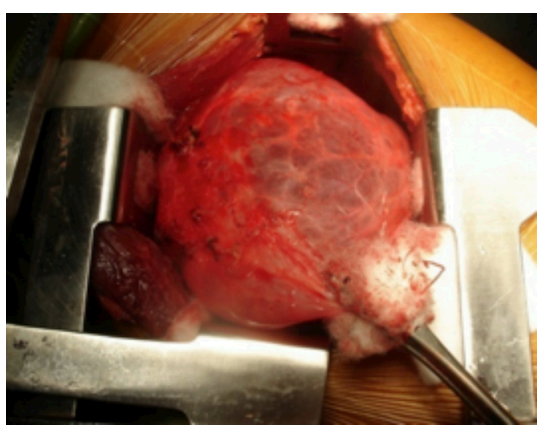

1 C. Lobectomía 


\section{SECUESTRO PULMONAR (SP)}

Es un sector del pulmón no funcionante sin comunicación con el árbol bronquial que recibe irrigación de la circulación sistémica. La etiología no ha sido completamente aclarada. Se clasifican en intralobar (SI) y extralobar (SE) con un radio de 3:1. Hay una tercera forma más rara, Ilamada MPC del intestino anterior en el que un pulmón anormal está conectado al tracto gastrointestinal (6). El SI presenta tejido pulmonar anómalo integrado al resto del pulmón normal con el que comparte revestimiento pleural visceral. El drenaje venoso es hacia las venas pulmonares 0 un colector venoso. Se localiza en los segmentos basales de los lóbulos inferiores, especialmente el izquierdo (80\%) en estrecha proximidad al surco costofrénico. En el SE (o lóbulo supernumerario) el parénquima pulmonar anormal está separado del tejido pulmonar normal por un revestimiento pleural propio. Generalmente está asociado a otras malformaciones como hernia diafragmática y puede ser un hallazgo en cirugías abdominales 0 cirugías de hernias o eventraciones diafragmáticas. El retorno venoso es hacia vena ácigos o venas cavas. El 15\% están situados debajo del diafragma (15).

Ambos secuestros reciben irrigación desde la aorta torácica o abdominal. En aproximadamente el 15\% de los casos la irrigación es proporcionada por una arteria sistémica diferente (15).

En el SE se observan bronquios y bronquiolos irregulares y alvéolos y vasos linfáticos subpleurales dilatados. El SI presenta múltiples quistes de diversos tamaños con parénquima pulmonar con tejido inflamatorio y fibrosis con vestigios de bronquios y los bronquiolos. En un alto porcentaje se asocian a MAQ (8).

Más del $50 \%$ de los SI se diagnostican en la adolescencia. Los pacientes pueden desarrollar neumonía recurrente y hemoptisis o permanecer asintomáticos y ser diagnosticado incidentalmente $(6,15)$.

En el período prenatal suele ser difícil diagnosticar el SE por ecografía porque se confunde con un pulmón normal porque Ios tejidos tienen características similares. El SI puede ser diagnosticado erróneamente como MAQ. La resonancia nuclear magnética (RNM) prenatal clarifica el diagnóstico.

La RXT muestra imágenes de condensación triangular persistente en una de las bases que se confirma con una angiotomografía donde se pone de manifiesto el vaso anómalo así como eventuales malformaciones asociadas. La resolución de ambos secuestros es siempre quirúrgica. En algunos casos previamente a la cirugía puede efectuarse embolización del vaso anómalo (Figura 2).
Figura $\mathrm{N}^{\circ} 2$

Secuestro pulmonar asociado a malformación adenomatosa quística

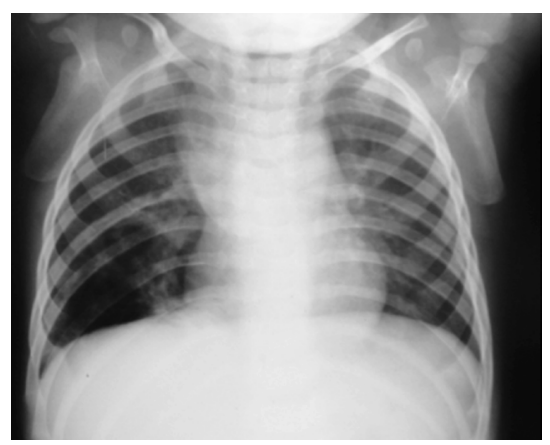

2 A. Radiografía de tórax: imagen quística en lóbulo inferior derecho.

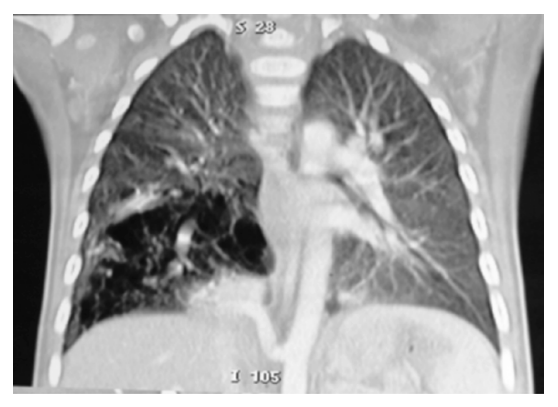

2 B. Tomografía computarizada: muestra irrigación a partir de la arteria aorta abdominal.

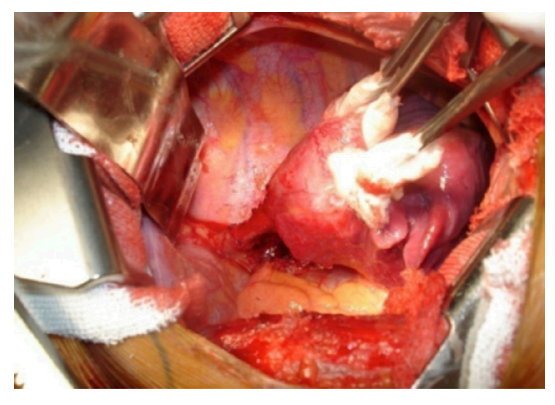

2 C. Lobectomía: se aprecia el pedículo vascular anómalo.

\section{ENFISEMA LOBAR CONGÉNITO (ELC)}

Es una MPC rara. Su incidencia es de 1:20.000 a 1:30.000 nacimientos (16). Se trata de una sobredistensión de uno 0 más lóbulos pulmonares. El lóbulo hiperinsuflado comprime estructuras adyacentes a medida que se distiende. Las causas principales son la deficiencia del desarrollo del cartílago bronquial $(25 \%)$ y la obstrucción bronquial de tipo valvular generalmente causada por estenosis bronquial idiopática (25\%). En el resto de los casos se desconoce el origen (6). El ELC afecta el lóbulo superior izquierdo (42\%), lóbulo medio (35\%), lóbulo superior derecho (21\%) y lóbulos inferiores (2\%). 
La edad de diagnóstico depende de la severidad de los síntomas respiratorios. Un tercio de los pacientes presenta síntomas durante el primer mes de vida y un $50 \%$ después de los 6 meses de vida. Un pequeño porcentaje puede permanecer asintomático durante años. En el recién nacido el ELC con gran sobredistensión lobar constituye una emergencia, con requerimiento de una cirugía de urgencia. Se puede observar dificultad respiratoria severa y progresiva a medida que se distiende el lóbulo. Desde el primer hasta el $8^{\circ}$ mes de vida se puede presentar tos, taquipnea, sibilancias recurrentes y cianosis con el Ilanto con asimetría torácica e hipersonoridad y a partir de los 3 años episodios de obstrucción bronquial recurrente o constituir un hallazgo radiológico. Hasta un 10\% de los casos presentan malformaciones cardiacas asociadas.

En las RXT se observa hiperinsuflación del lóbulo con menor trama pulmonar acompañada de hernia pulmonar a través de la línea media y desplazamiento del mediastino con atelectasia del lóbulo remanente. La ventilación/perfusión pulmonar permite determinar si el problema está en el lóbulo distendido o si se encuentra en el pulmón contralateral. La TC complementa el diagnóstico. La endoscopia descarta otras casusas y confirma las causas intrínsecas del ELC. El diagnóstico diferencial se realiza con el neumotórax, neumatoceles, hipoplasia pulmonar y con el enfisema adquirido (Figura 3).

En el período prenatal, el ELC se puede diagnosticar mediante una ecografía. Se han reportado casos con reducción de volumen espontáneo y resolución intraútero (17). El lóbulo afectado puede aparecer opacificado inicialmente debido a la retención de líquido amniótico.

La histología del ELC muestra dilatación alveolar leve, sin malformación o destrucción de los septos alveolares. En algunos casos el número de alvéolos puede aumentar (pulmón o lóbulo hiperplásico).

En los pacientes con síntomas, de acuerdo al grado de compromiso se efectúa cirugía de urgencia o programada, siendo la lobectomía (por toracotomía o videoasistida) el tratamiento de elección. La evolución post quirúrgica es favorable y sin secuelas (16).

\section{Figura $\mathrm{N}^{\mathbf{0} 3}$}

Enfisema lobar congénito.
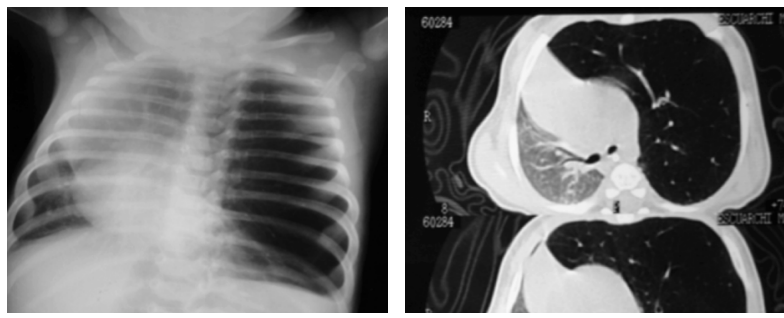

Se observa hiperinsuflacion del lóbulo superior izquierdo. Obsérvese el desplazamiento del mediastino hacia el lado contralateral con atelectasia del lóbulo inferior izquierdo. $3 \mathbf{A}$. Radiografía de tórax.

3 B. Tomografía computada de tórax.

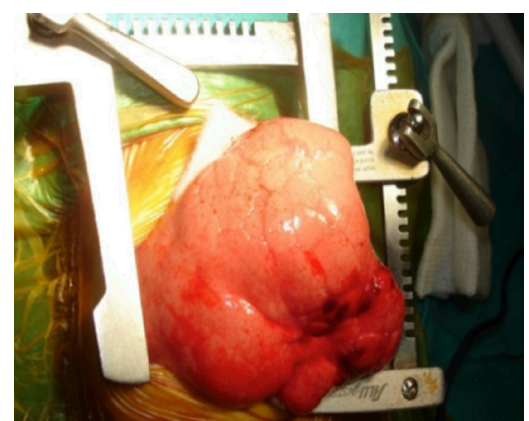

3 C. Cirugía: protrusión del lóbulo sobredistendido a través de la incisión.

\section{QUISTE BRONCOGÉNICO (QB)}

Es una formación quística unilocular, redondeada de entre 2 y $10 \mathrm{~cm}$ en relación con el árbol traqueobronquial. En general no tienen comunicación con vía aérea. Poseen una delgada pared de epitelio pseudoestratificado ciliado con contenido mucoide que puede contener glándulas bronquiales, musculo liso y cartílago. De acuerdo a su localización se clasifican como intrapulmonares o mediastinales. Son más frecuentes a nivel de la carina (50\%). Se pueden asociar a otras malformaciones (MAQ, ELC) (6).

Suelen ser asintomáticos, siendo un hallazgo casual en la RXT o presentar síntomas de compresión de la vía aérea o infecciones recurrentes cuando tiene comunicación con la vía aérea.

En la RXT muestra una imagen redondeada de bordes definidos con contenido aéreo o sólido según tenga comunicación con la vía aérea. Los quistes ubicados entre el esófago y la tráquea evidencian un desplazamiento posterior del esófago y anterior de la tráquea. Las imágenes deben confirmarse con TC. En el diagnóstico diferencial deben descartarse MAQ, quiste hidatídico, quiste simple y abscesos pulmonares. El tratamiento es quirúrgico con la exéresis del quiste y eventualmente lobectomía si el QB es intrapulmonar (Figura 4)

\section{Figura $\mathrm{N}^{\circ} 4$}

Quiste broncogénico.
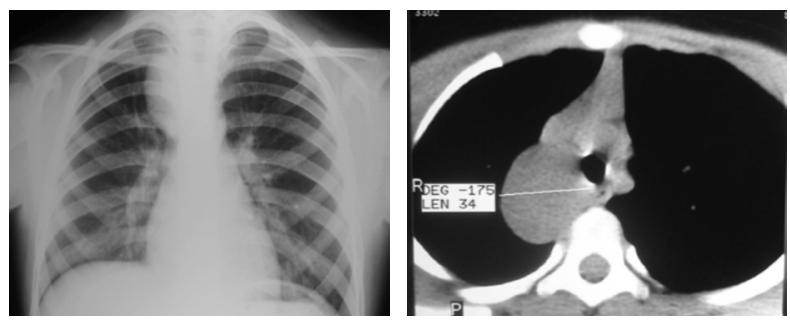

Imagen paramediastinal derecha correspondiente a un quiste broncogénico.

4 A. Radiografía de tórax.

4 B. Tomografía computada de tórax. 


\section{TRATAMIENTO}

\section{Alternativas de tratamiento prenatal}

En los últimos años hubo grandes avances en el tratamiento prenatal de las MCP y la mayoría de los embarazos con un feto afectado evolucionan favorablemente $(12,18-21)$.

La propuesta de tratamiento prenatal incluye estrategias curativas o paliativas para numerosas malformaciones. El tratamiento está orientado a resolver especialmente las complicaciones de los casos de malformaciones con quistes grandes que pueden desarrollar hidrops fetal.

Entre las alternativas se encuentra la aspiración repetida del quiste aunque puede ser una medida temporal ya que suele haber re-expansión del quiste dentro de las 24 a 72 hs. La derivación toracoamniótica también se ha propuesto para drenar quistes pulmonares grandes e hidrotórax asociados a secuestros (19-21).

Para las lesiones sólidas se ha propuesto la lobectomía fetal. También se han intentado métodos menos invasivos, como radiofrecuencia o ablación con láser con éxito limitado. La escleroterapia percutánea guiada por ultrasonido también ha sido utilizada en series de pocos pacientes (19-21).

Otra alternativa es el procedimiento quirúrgico que se realiza durante el parto por cesárea mientras el niño aún está conectado a la madre por el cordón umbilical, designado terapia ex utero-intrapartum (EXIT). Tiene una tasa de supervivencia de casi el $90 \%$ mientras que la cirugía abierta que se lleva a cabo en el período fetal tiene una tasa de supervivencia de aproximadamente el $50 \%$ (21).

La administración materna prenatal de betametasona también se planteó como tratamiento de los quistes grandes con riesgo de hidrops con resultados variables.

Todos estos tratamientos prenatales requieren de un equipo médico adecuadamente formado y equipado, y están limitados a centros de alta complejidad.

\section{Tratamiento postnatal. Momento oportuno de la cirugía}

Los niños que presentan síntomas precozmente tienen indicación ineludible de resección quirúrgica en el momento de aparición de los síntomas independientemente de la edad. La recurrencia de infecciones condiciona una resección quirúrgica más dificultosa y el riesgo de infección es de 10\% a 30\% en el primer año de vida (22). La posibilidad de malignización de estas lesiones es otro aspecto a considerar (8).

Existe controversia sobre la indicación quirúrgica de los casos con diagnóstico prenatal que son asintomáticos luego del nacimiento. El manejo es discutido debido a que el pronóstico de las MPC es en gran parte desconocido, si bien se estima que entre el $20 \%$ al $86 \%$ de los pacientes asintomáticos desarrollará síntomas a los 6 meses de edad (23).

Los argumentos a favor de la observación están basados en estudios observacionales retrospectivos. La involución espontánea intraútero de algunas MPC durante el período prenatal ha fortalecido esta posición (24). Los argumentos a favor 0 en contra la cirugía se enumeran laTabla1 $(19,20)$.

En todos los niños asintomáticos con diagnóstico prenatal de MPC debe efectuarse una RXT al nacimiento $(70 \%$ de sensibilidad). Si es normal deberá efectuarse una TC 0 eventualmente una RMN entre los 4-6 meses para confirmar o descartar el hallazgo (100\% de sensibilidad). La cirugía se recomienda durante el primer año de vida, entre los 6 y 9 meses. La resección quirúrgica en este período permite un crecimiento compensatorio pulmonar. No debe minimizarse el estrés psicológico de la familia y el incremento de radiaciones debido a las tomografías solicitadas durante un seguimiento prolongado (25).

Ante la decisión quirúrgica tomada se debe considerar la extirpación de manera radical mediante cirugía convencional o por toracoscopía videoasistida. A esta edad la evolución postoperatoria es favorable. Las tasas de complicaciones postquirúrgicas están entre el $6 \%$ y el $9 \%$ con baja mortalidad (8). El crecimiento pulmonar compensatorio permite una recuperación morfológica y funcional completa (26).

\section{Tabla N ${ }^{0} 1$}

Argumentos a favor 0 en contra de la intervención quirúrgica de las MPC asintomáticas

\section{Argumentos a favor de la observación}

- Hallazgo incidental en paciente asintomático

- Desconocimiento de la historia natural de las MPC

- Posibilidad de regresión

- Riesgos relacionados al procedimiento quirúrgico

\section{Argumentos a favor de la intervención quirúrgica}

- Alto riesgo de infecciones recurrentes, que complica la cirugía

- Malignización de las lesiones (indistinguibles por imágenes)

- Riesgo de neumotórax o aumento súbito de volumen de los quistes

- Recuperación rápida post-quirúrgica en niños pequeños asintomáticos

- Crecimiento compensatorio pulmonar

\section{CONCLUSIONES (PUNTOS CLAVE)}

- Las MPC tendrían un origen común.

- Aunque haya ocurrido resolución in útero todo recién nacido debe ser estudiado.

- Considerar la presencia de una MPC en los casos de neumonia recurrente localizada.

- Distinguir lesiones quísticas de hernia diafragmática.

- Evaluar conducta quirúrgica vs conservadora en forma individual. 
El autor declara no tener conflicto de intereses.

Agradecimientos: las imágenes presentadas en la presente revisión fueron cedidas por el Jefe de Servicio de Cirugía del Hospital Garrahan, Dr. Marcelo Barrenechea.

\section{REFERENCIAS}

1. Groenman F, Sharon Unger S, Post M. The Molecular Basis for Abnormal Human Lung Development. Biol Neonate 2005;87:164-177

2. Langston C. New concepts in the pathology of congenital lung malformations. Semin Pediatr Surg. 2003;12:17-37

3. Newman B. Congenital bronchopulmonary foregut malformations: concepts and controversies. Pediatr Radiol. 2006;36: 773-791

4. Peranteau WH, Merchant AM, Hedrick HL, Liechty KW, Howell LJ, Flake AW, Wilson RD, Johnson MP, Bebbington MW, Adzick NS. Prenatal course and postnatal management of peripheral bronchial atresia: association with congenital cystic adenomatoid malformation of the lung. Fetal Diagn Ther 2008;24: 190-6

5. Bush A. Prenatal presentation and postnatal management of congenital thoracic malformations. Early Human Development. 2009;85:679-84

6. Andrade C, da Costa Ferreira H, Fisher G. Congenital lung malformations. J Bras Pneumol. 2011;37:259-271

7. Papagiannopoulos K, Hughes S, Nicholson AG, Goldstraw P. Cystic lung lesions in the pediatric and adult population: surgical experience at the Brompton Hospital. Ann Thorac Surg. 2002;73:1594-8

8. Giubergia V, Barrenechea $M$, Siminovich $M$, Pena HG, Murtagh P. Congenital cystic adenomatoid malformation: clinical features, pathological concepts and management in 172 cases. J Pediatr (Rio J) 2012;88:143-8

9. Chin KY, Tang MY. Congenital cystic adenomatoid malformation of one lobe of a lung with general anasarca. Arch Pathol 1949; 48:221-229

10. Stocker JT. Congenital pulmonary airway malformation: a new name and an expanded classification of congenital cystic adenomatoid malformation of the lung. Hystopathology 2002;41(suppl 2):424-58 11. Azizkhan RG, Crombleholme TM. Congenital cystic lung disease: contemporary antenatal and postnatal management. Pediatr Surg Int 2008 Jun;24(6):643-57
12. Epelman M, Kreiger PA, Servaes S, Victoria T, Hellinger JC. Current Imaging of Prenatally Diagnosed Congenital Lung Lesions. Semin Ultrasound CT MR 2010;31:141-57

13. Costa F, Kaganov H, O'Mahony E, Ng J, Fink AM, Palma-Dias R. Diagnosis of diaphragmatic hernia with associated congenital lung lesions: contribution of fetal MRI. Fetal Diagn Ther 2011;29:111-5

14. Butterworth SA, Blair GK. Postnatal spontaneous resolution of congenital cystic adenomatoid malformations. J Pediatr Surg. 2005;40:832-4

15. Corbett HJ, Humphrey GM. Pulmonary sequestration. Paediatr Respir Rev. 2004;5:59-68

16. Mei-Zahav M, Konen 0 , Manson D, Langer JC. Is congenital lobar emphysema a surgical disease? J Pediatr Surg. 2006;41:1058-61

17. Nayar PM, Thakral CL, Sajwani MJ. Congenital lobar emphysema and sequestration-treatment by embolization. Pediatr Surg Int. 2005;21:727-9

18. Laberge JM, Puligandla P, Flageole H. Asymptomatic congenital lung malformations Semin Pediatr Surg 2005;14:16-33

19. Puligandla P, Laberge JM. Congenital Lung Lesions Clin Perinatol. 2012; 39:331-347

20. Mann S, Wilson RD, Bebbington MW, Adzick NS, Johnson MP. Antenatal diagnosis and management of congenital cystic adenomatoid malformation. Semin Fetal Neonatal Med 2007;12:477-81

21. Adzick NS. Management of Fetal Lung Lesions. Clin Perinatol 2009;36(2):363-76

22. Pelizzo G, Barbi E, Codrich D, Lembo MA, Zennaro F, Bussani R, Schleef J. Chronic inflammation in congenital cysticadenomatoid malformations. An underestimated risk factor? J Pediatr Surg 2009;44:616-619

23. Conforti A, Aloi I, Trucchi A, Morini F, Nahom A, Inserra A, Bagolan P. Asymptomatic congenital cystic adenomatoid malformation of the lung: is it time to operate? J Thorac Cardiovasc Surg 2009;138:826-30

24. Jaffe A, Chitty LS. Congenital cystic adenomatoid malformations may not require surgical intervention. Arch Dis Child Fetal Neonatal Ed 2006;91:F464

25. Raychaudhuri P, Pasupati A, James A, Whitehead B, Kumar R. Prospective study of antenatally diagnosed congenital cystic adenomatoid malformations. Pediatr Surg Int 2011;27:1159-64

26. Beres A, Aspirot A, Paris C, Berube D, Bouchard S, Laberge JM, Lands LC, Puligandla P. A contemporary evaluation of pulmonary function in children undergoing lung resection in infancy. J Pediatr Surg 2011;46:829-32 